\title{
Matched related donor hematopoietic stem cell transplantation protocol for patients with primary immunodeficiency
}

\author{
Vy Hong-Diep Kim, for the SickKids Immunodeficiency Transplant Program
}

The exact role of factors affecting the outcome of hematopoietic stem cell transplantation (HSCT) are difficult to assess. Some determinants like genotype and pathway affected are inherently hard to evaluate in rare disorders, such as severe combined immunodeficiency (SCID). Comparison of outcomes even within an institution has been challenging because of the use of multiple inconsistent protocols. To eliminate this possibility in our cohort of patients, we have established detailed protocols for various indications. For patients with primary immunodeficiency (PID) in whom residual function is present and who have a human leukocyte antigen (HLA) matched related donor (MRD), we use the HSCT protocol that has previously been published for HLA matched unrelated donors (Kim 2016). Here, we present a HSCT protocol for patients with SCID who do not have $\mathrm{T}$ cells or NK cells, and who have a MRD (Table 1). We hope this approach could be beneficial, especially to new faculty and budding HSCT programs.

Statement of novelty: This report describes the protocols for HSCT using matched related donors for patients with primary immunodeficiency at our institution.
Division of Immunology and Allergy, Department of Paediatrics, The Hospital for Sick Children, University of Toronto, Toronto, ON
Submitted 6 February 2017

Accepted 6 March 2017

Available online 7 March 2017

LymphoSign Journal 4:42-44 (2017)

dx.doi.org/10.14785/lymphosign-2017-0002 
Table 1: MRD HSCT protocol for patients with SCID with no residual function.

\begin{tabular}{|c|c|}
\hline Day -4 & $\begin{array}{l}\text { Admit under reverse isolation (Reid and Courtney 2015) } \\
\text { Standing orders } \\
\text { Irradiate all blood products and use CMV negative blood products } \\
\text { Daily weight } \\
\text { Bacti-stat bath daily } \\
\text { Transfuse to keep hemoglobin }>70 \mathrm{~g} / \mathrm{L} \text {, platelets }>20 \times 10^{9} / \mathrm{L} \\
\text { Discontinue breast feeding or irradiate breast milk } \\
\text { Standing bloodwork orders } \\
\text { Send blood to Molecular Genetics lab for as Pre-HSCT recipient sample for donor chimerism } \\
\text { CBC, Na, K, Ca, P, Mg, glucose, urea, creatinine, ALT, AST, GGT, conjugated bilirubin, and unconjugated } \\
\text { bilirubin daily } \\
\text { Differential qMonday, Wednesday, Friday } \\
\text { INR, PTT, NH } 3 \text {, amylase, total protein, albumin, IgG level qMonday } \\
\text { Urine total protein and albumin/creatinine ratio qWednesday if no hematuria } \\
\text { Lipid levels twice per week while on TPN } \\
\text { CMV, EBV by PCR, quantitative EBV by PCR, adenovirus by PCR qMonday } \\
\text { Standing medication orders } \\
\text { IVIG (0.6 g/kg/dose) IV over } 2 \text { h, once weekly if CMV positive or if IgG < } 6 \text { g/L } \\
\text { Sodium bicarbonate mouthwash } 5 \mathrm{~mL} \text { apply with cloth/toothbrush to teeth and gums QID for mouth care } \\
\text { Eucerin }{ }^{\circledR} \text { cream apply to skin daily after bath and PRN } \\
\text { Discontinue previous cotrimoxazole order } \\
\text { Cotrimoxazole (5 mg TMP/kg/dose) PO daily } \times 3 \mathrm{~d} \\
\text { Start fluconazole (5 mg/kg/dose, max } 400 \text { mg/dose) PO/IV once daily } \\
\text { Consider ganciclovir ( } 5 \text { mg/kg/dose) IV BID for CMV positive recipient } \\
\text { Start acyclovir ( } 250 \text { mg/m²/dose) IV q } 8 \text { h for HSV prophylaxis (if not receiving ganciclovir) } \\
\text { Consider amphotericin for treatment and possibly prophylaxis of fungal infection until Day } 0\end{array}$ \\
\hline Day -3 & $\begin{array}{l}\text { Start cyclosporine A ( } 1.5 \mathrm{mg} / \mathrm{kg} / \mathrm{dose}) \text { IV q12 h over } 2 \mathrm{~h} \\
\text { Standing order: Draw pre cyclosporine A level qMonday, Wednesday, Friday (target level: } 150-200 \mathrm{mcg} / \mathrm{L})\end{array}$ \\
\hline Day -2 & Continue cyclosporine A (dose based on cyclosporine A level) \\
\hline Day -1 & Discontinue ganciclovir if used \\
\hline Day 0 & $\begin{array}{l}\text { IV fluids at maintenance } \\
\text { Consider starting methylprednisolone ( } 1 \mathrm{mg} / \mathrm{kg} / \mathrm{dose} \text { ) IV q12 } \mathrm{h} \\
\text { Start pentamidine ( } 4 \mathrm{mg} / \mathrm{kg} / \mathrm{dose} \text { ) IV q2 wk until cotrimoxazole restarts } \\
\text { Continue cyclosporine A (dose based on cyclosporine A level) } \\
\text { Hematopoietic progenitor cell infusion orders } \\
\text { IV hydration ( } 125 \mathrm{~mL} / \mathrm{m}^{2} / \mathrm{h} \text { ) for } 2 \mathrm{~h} \text { pre and } 4 \mathrm{~h} \text { post HPC infusion, then resume previous hydration order } \\
\text { Ensure anaphylaxis kit available and MD on site } \\
\text { Continuous ECG monitoring and oxygen saturation monitoring } \\
\text { Vital signs (temperature, HR, RR, BP) q15 min } \\
\text { Diphenhydramine }(1 \mathrm{mg} / \mathrm{kg} / \text { dose) IV, give once } 30 \text { min prior to HPC infusion } \\
\text { Acetaminophen }(10 \mathrm{mg} / \mathrm{kg} / \mathrm{dose}) \mathrm{PO} \text {, give once } 30 \text { min prior to HPC infusion } \\
\text { Meperidine }(1 \mathrm{mg} / \mathrm{kg} / \mathrm{dose}) \text { IV q2 } \mathrm{h} \text { PRN rigors during HPC infusion } \\
\text { Infuse HPC } 3-5 \times 10^{8} \mathrm{TNC} / \mathrm{kg} \text { (reduce volume for small recipients, RBC reduce for ABO incompatibility) }\end{array}$ \\
\hline Day +28 & $\begin{array}{l}\text { Discontinue fluconazole } \\
\text { Discontinue acyclovir on Day }+28 \text { or discharge, whichever comes sooner } \\
\text { Start cotrimoxazole }\left(5 \mathrm{mg} / \mathrm{kg} / \mathrm{d} \text { ) TMP PO daily } 3 \mathrm{~d} \text { per week when ANC }>0.5 \times 10^{9} / \mathrm{L} \text { and platelets }>50 \times 10^{9} / \mathrm{L}\right. \\
\text { Or continue pentamidine }(4 \mathrm{mg} / \mathrm{kg} / \mathrm{dose}) \text { IV q } 2 \mathrm{wk} \text { if cotrimoxazole delayed } \\
\text { Or dapsone }(2 \mathrm{mg} / \mathrm{kg} / \mathrm{dose}) \mathrm{PO} \text { daily } \\
\text { Restart ganciclovir }(5 \mathrm{mg} / \mathrm{kg} / \mathrm{dose}) \text { IV daily for CMV positive recipient or CMV positive donor }\end{array}$ \\
\hline
\end{tabular}

Abbreviations: ALT, alanine aminotransferase; ANC, absolute neutrophil count; AST, aspartate aminotransferase; Ca, calcium; CBC, complete blood counts; CMV, cytomegalovirus; EBV, Epstein-Barr virus; GGT, gamma-glutamyl transferase; INR, international normalized ratio; IVIG, intravenous immune globulin; $\mathrm{K}$, potassium; Mg, magnesium; $\mathrm{Na}$, sodium; $\mathrm{NH}_{3}$, ammonia; $\mathrm{P}$, phosphate; PCR, polymerase chain reaction; PTT, partial thromboplastin time; TMP, trimethoprim; TPN, total parenteral nutrition; TNC, total nucleated cells. 


\section{Acknowledgements}

The author would like to thank past and current members of the Division of Immunology/Allergy at our institution for their invaluable contributions to the establishment of our HSCT protocols for patients with SCID and PID and to the outstanding care of our patients.

\section{REFERENCES}

Kim, V.H. 2016. A protocol for matched unrelated donor hematopoietic stem cell transplantations for severe combined immunodeficiency. LymphoSign J. 3:41-45. doi: 10.14785/lpsn-2015-0015.

Reid, B., and Courtney, S. 2015. Isolation protocol for patients with severe combined immune deficiency. LymphoSign J. 2:165-170. doi: 10.14785/lpsn-2015-0011. 